\title{
eNeuro
}

Research Article: New Research / Sensory and Motor Systems

\section{Faster repetition rate sharpens the cortical representation of echo streams in echolocating bats}

https://doi.org/10.1523/ENEURO.0410-21.2021

Cite as: eNeuro 2021; 10.1523/ENEURO.0410-21.2021

Received: 30 September 2021

Revised: 18 November 2021

Accepted: 23 November 2021

This Early Release article has been peer-reviewed and accepted, but has not been through the composition and copyediting processes. The final version may differ slightly in style or formatting and will contain links to any extended data.

Alerts: Sign up at www.eneuro.org/alerts to receive customized email alerts when the fully formatted version of this article is published.

Copyright (c) 2021 Macias et al.

This is an open-access article distributed under the terms of the Creative Commons Attribution 4.0 International license, which permits unrestricted use, distribution and reproduction in any medium provided that the original work is properly attributed. 
1 1. Title: Faster repetition rate sharpens the cortical representation of echo streams in

2 echolocating bats.

3 2. Abbreviated Title: High repetition rate enhances objects representation

4 3. Author names: Silvio Macias ${ }^{\mathrm{a}}$, Kushal Bakshi ${ }^{\mathrm{b}}$, Michael Smotherman ${ }^{\mathrm{a}, \mathrm{b}}$

\section{Affiliations:}

$6 \quad{ }^{\mathrm{a}}$ Department of Biology, Texas A\&M University, College Station, Texas, USA.

$7 \quad{ }^{\mathrm{b}}$ Institute for Neuroscience, Texas A\&M University, College Station, Texas, USA.

8 4. Author contribution:

9 S.M., K.B. and M.S. designed research;

10 S.M. and K.B. performed researched;

11 S.M. analyzed data;

12 S.M., K.B. and M.S. wrote the paper.

\section{Correspondence should be addressed to:}

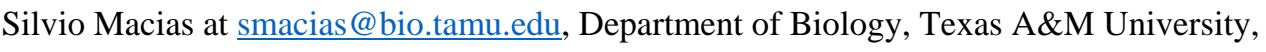
College Station, Texas, USA.

6. Number of figures: 7

7. Number of tables: 0

\section{Number of multimedia: 0}

9. Number of extended data figures/tables: 0 
10. Number of words for abstract (250 words maximum): 183 words

21 11. Number of words for Significance Statement (120 words maximum): 111 words

22 12. Number of words for Introduction ( $\mathbf{7 5 0}$ words maximum): 726 words

13. Number of words for Discussion (3000 words maximum): 966 words

14. Acknowledgments

25 We thank Barbara Earnest for her assistance with the animals and facilities.

\section{Conflict of interest:}

27 The authors declare no competing financial interests

\section{Funding sources:}

29 This research was funded by the US Office of Naval Research (grant \#ONRN00014-17-1-2736). 


\section{Abstract}

2 There is consensus that primary auditory cortex utilizes a combination of rate codes and temporally precise population codes to represent discreet auditory objects. During the response

4 to auditory streams, forward suppression constrains cortical rate coding strategies, but it may also be well-positioned to enhance temporal coding strategies that rely upon synchronized firing across neural ensembles. Here, we exploited the rapid temporal dynamics of bat echolocation to investigate how forward suppression modulates the cortical ensemble representation of complex acoustic signals embedded in echo streams. We recorded from auditory cortex of anesthetized free-tailed bats while stimulating the auditory system with naturalistic biosonar pulse-echo sequences covering a range of pulse emission rates. As expected, increasing pulse repetition rate significantly reduced the number of spikes per echo stimulus, but it also increased spike timing precision and doubled the information gain. This increased spike-timing precision translated into more robust inter-neuronal synchronization patterns with $>10 \mathrm{~dB}$ higher signal-to-noise ratios at the ensemble level. We propose that forward suppression dynamically mediates a trade-off

\section{Significance Statement}

Auditory cortical neurons are unable to follow trains of sounds with repetition rates higher than roughly $15 \mathrm{~Hz}$. The dynamics and synaptic mechanisms responsible for this forward suppression are well known. However, their functional consequences on the representation of sounds remain unknown. We evaluated the effects of forward suppression on both rate and temporal coding of 
synchronization-based time codes and thereby enhance the cortical spatiotemporal representation of natural sounds at ecologically relevant rates. Such increases in neuronal synchrony are consistent with emerging theories of how sound spectral envelopes are encoded, with important implications for music and speech processing.

\section{Introduction}

The responses of auditory cortical neurons to preferred sounds decrease over time with repeated stimulation at high rates. This forward suppression of neural activity is evident when one sound diminishes responses to subsequent similar sounds for tens to hundreds of milliseconds (Creutzfeldt et al., 1980; Eggermont, 1992; Brosch and Schreiner, 1997). This widespread phenomenon shapes the neural representation of ongoing complex sound sequences in such ways as preventing individual neurons from faithfully following sound trains faster than about $15 \mathrm{~Hz}$ (Eggermont, 1992). The temporal dynamics of forward suppression are now well described in many species, and recent studies have identified its cellular and synaptic origins (Wehr and Zador, 2005; Bayazitov et al., 2013). However, the theoretical consequences of forward suppression on the cortical representation of sounds remain speculative.

Complex sounds are composed of acoustic features that are independently encoded by the ascending auditory system and subsequently integrated by cortical networks that reconstruct a percept of the complete sound. This is accomplished through a combination of rate coding and time coding strategies in auditory cortex (Nelken et al., 2005). Rate coding captures feature dynamics by the number of evoked spikes per stimulus, while time codes track feature parameters based on relative spike timing across neural ensembles. Forward suppression sharply compresses the dynamic range of spike rates and thereby constrains rate coding (Yin et al., 2011; 
Malone et al., 2015; Hörpel and Firzlaff, 2019), but its impacts on spike timing or temporal coding schemes are less clear (Schnupp et al., 2006; Yin et al., 2011; Ince et al., 2013). Although there is extensive literature about the use of spike rate to encode temporal parameters, like duration or delay tuning, the bat auditory cortex also uses time coding strategies to cope with the rapid and temporally precise nature of echolocation (Suga, 1989). We took advantage of this to measure the effects of forward suppression on the cortical representation of complex sounds within the context of a computational model of information coding in auditory cortex (Ming et al., 2021).

The bat auditory system is adapted to analyze biosonar echoes (Suga, 1989), which are typically 2-3 ms broadband, downward frequency-modulated sweeps. When an echolocation pulse is reflected off of an irregularly shaped surface, multiple overlapping echoes convolve into a single sound endowed with a complex interference pattern of spectral peaks and notches (Simmons et al., 1974). Echo spectral notch patterns are therefore unique to each target, and bats can reconstruct an internal representation of a target's shape based on these fine spectral details (Schmidt, 1988; Simmons and Chen, 1989; Schmidt, 1992; Ming et al., 2021). The bat auditory cortex contains neurons that preferentially respond with greater spike rates to the presence of unique spectral interference patterns (Sanderson and Simmons, 2002; Firzlaff and Schuller, 2007). However, we recently reported evidence that the cortical representation of target shape may benefit more from the amount of information carried by the spike times, specifically the first spike latency of the response of the individual neurons of the bat primary auditory cortex.

Theoretically, the observed changes in the response latency of the neurons tuned to the frequency of the notches, usually in the range of 5-8 ms, disrupt or alter the sequential activation along the tonotopic axis of the primary auditory cortex, which leads to an increase of the spike synchrony 
between neurons tuned to different characteristic frequencies (Macias et al., 2020). This result in a cortical spatiotemporal spike synchronization pattern that should be unique for each target. Independently derived computational models also predict that bat auditory cortex relies upon a precise spike-time dependent synchronization network to reconstruct echoes and classify their source (Saillant et al., 1993; Ming et al., 2021). There is evidence that forward suppression contribute to a sharper rate coding of echo delay (Beetz et al., 2016). Here, we evaluated how forward suppression affects the rate and time coding of target shape in the primary auditory cortex of the echolocating bat. We examined how the reduced firing of the cortical neurons due to the suppression affects the spike synchronization patterns obtained for different targets. In addition, we assessed how forward suppression contributes to the amount of information carried by the changes in the response latency. The results show that forward suppression has the potential to greatly facilitate synchronization-based time codes and thereby enhance the neural representation of natural sounds at ecologically relevant rates.

\section{Materials and Methods}

\section{Animals}

We performed electrophysiological recordings in the primary auditory cortex (A1) of four adult (one female, three males) Mexican free-tailed bats, Tadarida brasiliensis. Bats were group housed indoors in an artificial habitat with a reversed light cycle. All animal experimental procedures were conducted in accordance with the National Institutes of Health's Guide for the Care and Use of Laboratory Animals and were approved by the Institutional Animal Care and Use Committee (IACUC Animal Use Protocol \#: 2017-0163D). 


\section{Surgical procedures}

Animals were anesthetized with a solution of sodium pentobarbital $(80 \mathrm{mg} / \mathrm{kg})$ and positioned within a custom-built stereotaxic apparatus. Status of anesthesia was monitored by monitoring breathing and ear twitch reflexes and maintained at a surgical plane with supplementary doses as needed. Body temperature was maintained within normal ranges using a heating lamp. The skin and temporal muscles overlying the skull were cut and removed and a custom-fabricated post was attached to the bone at the midline using cyanoacrylate gel. A craniotomy $(\sim 2 \times 2 \mathrm{~mm})$ was made using a scalpel blade to expose the left auditory cortex.

\section{Acoustic stimuli}

Acoustic stimuli were digitally synthesized and controlled using a custom-written program in MatLab (R2018a, MathWorks, Natick, MA, USA). Sounds were generated at a sampling rate of $250 \mathrm{kHz}$ with a National Instruments card (NI USB-6356, National Instruments Co, Austin, TX, USA). The audio signal was transferred to an audio amplifier (SONY, STR-DE197, NY, USA) and broadcast to the bat with a calibrated ribbon tweeter loudspeaker (Dayton Audio, PTMini-6, $\mathrm{OH}, \mathrm{USA}$ ) centered $10 \mathrm{~cm}$ directly in front of the head. The calibration curve was obtained with a Brüel and Kjaer sound recording system (1/4-inch Microphone 4135, Microphone Preamplifier 2670, Brüel and Kjaer, Naerum, Denmark) connected to a conditioning microphone amplifier (Nexus 2690, Brüel and Kjaer, Naerum, Denmark).

To measure the frequency response area, we presented the animal with a pseudorandomized series of pure tones (10 ms duration, $0.5 \mathrm{~ms}$ rise/fall time) at different sound pressure levels (step size $10 \mathrm{~dB}$, range $20-80 \mathrm{~dB}$ SPL) and frequencies (step size $5 \mathrm{kHz}$, range $10-80 \mathrm{kHz}$ ). Each frequency-level combination was presented 5 times at an interval of $300 \mathrm{~ms}$. We tested the effect of the temporal arrangement on the cortical representation of the object surfaces. To do this, we 
ensonified a flat surface and two sandpapers of different grit sizes (60 and 150) with a $3 \mathrm{~ms}$ downward FM sweeping between 20 and $70 \mathrm{kHz}$ through an ultrasound speaker (Dayton Audio, PTMini-6, OH, USA) and recorded the returning echoes with a microphone (Brüel and Kjaer, Naerum, Denmark, 1/4-inch Microphone 4135, Microphone Preamplifier 2670) located above the speaker. Using recorded the pulses-echoes, we built with sequences of different repetition rates $(10,12$ and $15 \mathrm{~Hz})$ for each surface.

\section{Electrophysiological recordings}

Experiments were performed in a custom-built sound-attenuating anechoic chamber.

Anesthetized bats were placed in a body mold made of soft plastic foam and the head was tightly affixed to the stereotaxic apparatus by a rod attached to a metal holder. Neuronal recordings were performed using silicon probes from Cambridge Neurotech $(16$ contacts $\times 2$ shanks per probe with $250 \mu \mathrm{m}$ between shanks and $50 \mu \mathrm{m}$ spacing between contact sites along each shank). Each shank had a thickness of $15 \mu \mathrm{m}$. Using a micromanipulator system (MX7600R, Siskiyou Corp., OR, USA), probes were positioned perpendicular to the pial surface based upon landmarks and stereotaxic coordinates, and then inserted slowly into the brain through the intact dura mater to a depth of approximately $900 \pm 50 \mu \mathrm{m}$ at the deepest contact point. Neuronal data were acquired with an OmniPlex D Neural Data Acquisition System recording system (Plexon Inc., Dallas, USA) at a sampling rate of $40 \mathrm{kHz}$ (per channel) and 16-bit precision. Synchronization between the neural recordings and acoustic stimulus broadcasts was achieved with a TTL pulse output from the National Instrument card and recorded on one of the analog channels of the Plexon data acquisition system. 


\section{Analysis of Neural Recordings}

Since we did not find differences in the frequency tuning, bandwidth of frequency response areas or directional selectivity to the FM sweep across cortical depth (Macias et al., 2019), in this study we only included data recorded at depths between 400 and $600 \mu \mathrm{m}$, corresponding to input layer IV. The raw signal was digitally bandpass-filtered offline (elliptic, 2nd order) between 500 and $3000 \mathrm{~Hz}$ to obtain the multiunit activity. Neural recordings were sorted following methods outlined by (Quiroga et al., 2004). The Wavelet transformation and the superparamagnetic clustering resulted in isolation of single-unit extracellular potentials that matched with qualitative assessments of spike waveforms and estimates of single-unit isolation based on spike refractory periods. Recordings with spike amplitudes lower than four times the amplitude of the recording background noise were not included in the data analysis. From the raster plots, representing the spike-time vs. the trial number, we measured the number of spikes in a window of $50 \mathrm{~ms}$ after the stimulus onset for each frequency-level combination to build the frequency response areas. In each frequency response area, we calculated the characteristic frequency $(\mathrm{CF}$, frequency eliciting the higher number of spikes at the lowest level). In the responses to the CF at $80 \mathrm{~dB}$ SPL, we measured the number of spikes and the mean first spike latency (mean FSL). Because some neurons showed some spontaneous firing, we calculated the mean FSL by measuring the time of the first spike after the post stimulus time histogram reached $25 \%$ of its peak. This minimized the influence of spontaneous activity spikes on the response times.

Blood vessels around the medial cerebral artery were very consistent from bat to bat. This allowed us to locate the A1 and use the vessels as reference points for stereotaxic measurements. For each bat, coordinates of the recording sites in relation to a branch of the median cerebral artery were measured using a calibrated micromanipulator (MX7600R, Siskiyou Corp.). All 
cortices were aligned together for the construction of composite maps using the branches and the median cerebral artery to determine the orientation of the ordinate axis of the bi-dimensional Cartesian space of analysis.

We calculated synchronization matrices using the response from the population of neurons (Macias et al., 2020) to the echoes from the flat and the two sandpapers surfaces. To build the synchronization matrices, we evaluated spike train synchrony using the spike-synchronization index (c), which quantifies the degree of synchrony from the relative number of quasisimultaneous appearance of spikes. We used SPIKY (Kreuz et al., 2015; Satuvuori et al., 2017), a Matlab (MathWorks, Natick, MA) written graphical user interface for monitoring synchrony between artificially simulated or experimentally recorded neuronal spike trains. Synchronization matrices were calculated using the spike trains elicited in response to the echoes from the different surfaces in all 165 neurons. A matrix was calculated for each trial and from that, we calculated the mean synchronization matrix for each echo.

We evaluate the effect of the forward suppression in the cortical synchronization patterns by calculating the signal to noise ratio (SNR) of the synchronization matrices, we considered each matrix as an image and used the matrix calculated in the response to the first pulse-echo pair as the reference. The SNR of each matrix can be expressed as follow:

$$
S N R=10 \log _{10}\left(\frac{x^{2}}{R}\right)
$$

where $\mathrm{x}$ is the matrix to evaluate and $\mathrm{R}$ is the reference matrix (Gonzalez and Woods, 2008).

We calculated mutual information (MI), that quantifies how well an ideal observer of neuronal responses can discriminate between the different stimuli, based on a single response trial 
177 can be expressed as follows:

$$
I(R ; S)=H(R)-H(R \mid S)
$$

178 179

180

while

$$
H(R \mid S)=-\sum_{s \in S} P(s) \sum_{r \in R} P(r \mid s) \log _{2}[P(r \mid s)]
$$

181

182

183

where $\mathrm{H}(\mathrm{R})$ is the response entropy (i.e. the total variability of the response distribution) and is calculated as

$$
H(R)=-\sum_{r \in R} P(r) \log [P(r)]
$$

is known as the "noise entropy" and represents the irreproducibility of the response given a stimulus. The probabilities $\mathrm{P}(\mathrm{r})$ and $\mathrm{P}(\mathrm{s})$ represent the probability of a particular response in $\mathrm{R}$, and the probability of a particular stimulus in $\mathrm{S}$, respectively, while $\mathrm{P}(\mathrm{r} \mid \mathrm{s})$ represents the conditional probability of a response $\mathrm{r}$ given a stimulus s. To calculate the neuronal responses (spike rate and mean FSL), we considered a time window of 0-60 ms after the stimulus onset. In calculating the information conveyed only by the spike rate, the response $r$ was computed as the number of spikes emitted in this time window on one trial. To study information conveyed by the FSL, we divided the spike trains into bins of $2 \mathrm{~ms}$. Both $\mathrm{P}(\mathrm{r})$ and $\mathrm{P}(\mathrm{s})$ depend on the assumptions made regarding how the response is quantified, and how the stimulus set is defined. Note that the units of MI are bits, given that the logarithm used for the calculations is of base 2. Each bit of information implies that an observer can reduce its uncertainty about the stimulus (based on the 
response) by a factor of 2 . All information analyses were conducted using the Information Breakdown Toolbox (ibTB) (Panzeri et al., 2001; Magri et al., 2009).

\section{Results}

\section{Creating naturalistic echo mimic stimuli to measure information coding in auditory cortex}

To investigate the effects of forward suppression on the temporal coding of echo spectral envelope, we first needed to create a set of echo mimic acoustic stimuli that we knew the bats could distinguish. Surface texture is an integral cue used by echolocating bats and dolphins for classifying an ensonified target, and previous work showed that bats rely on spectral notch patterns embedded in echoes to resolve textures (Habersetzer and Vogler, 1983; Schmidt, 1988; Simmons et al., 1990). Abrasive sandpapers are available as standardized textured surfaces that provided a convenient way to generate consistent, complex echo spectral patterns. Sandpaper coarseness is indicated by grit number, which is inversely related to the mean particle diameter of the abrasive coating. A two-alternative forced choice assay confirmed that free-tailed bats can discriminate between different sandpaper grits by echolocation (Smotherman et al., 2020), and based on this we selected two sandpaper grits with distinctive echo spectral features that the bats readily distinguished. To make the acoustic stimuli, we ensonified three different reflecting surfaces (a flat plexiglass surface, a 60 -grit surface [265 $\mu$ m mean particle diameter] and a 150 grit surface [92 $\mu \mathrm{m}$ mean particle diameter]) with an artificial downward FM sweep played through an ultrasonic loudspeaker while recording echoes with a microphone directly above the speaker. The sandpaper was always located at a distance of $80 \mathrm{~cm}$, which produced a fixed temporal separation of $5 \mathrm{~ms}$ between the pulse and the recorded echo. This ensured that there was no temporal overlapping between pulse and echo and that the spectral notches caused in the 
echo were caused only by the surface structure of the sandpaper. Figure 1 shows the oscillogram, spectrogram and the power spectra of the resulting echo mimic stimuli and their distinctive spectral notch patterns. For example, while the echo resulting from the flat surface shows no notches either in the spectrogram or the power spectra, the echo resulting from the 60 -grit surface shows a $40 \mathrm{~dB}$ amplitude notch at $30 \mathrm{kHz}$ and the echo from the 150 -grit surface had two noticeable amplitude notches at 35 and $45 \mathrm{kHz}$, as well as a decreased amplitude at frequencies higher than $50 \mathrm{kHz}$.

\section{Increasing pulse repetition rate suppresses firing rate but enhances spike precision}

Each stimulus pattern was presented as part of a pulse-echo combination delivered at three different repetition rates, 10, 12 and 15 per second. A $10 \mathrm{~Hz}$ sequence consisted of ten pulseecho combinations with a temporal separation of $100 \mathrm{~ms}$. The $12 \mathrm{~Hz}$ sequence included twelve pulse-echo combinations with $88 \mathrm{~ms}$ intervals and the $15 \mathrm{~Hz}$ had fifteen pairs separated by 66 ms. Theses repetition rates were chosen based on our previous behavioral studies of pulse emission rates of this species of bat in a stationary position and while flying. Both stationary and flying free-tailed bats normally emit sustained emission rates of 10-15 pulses per second when actively echolocating (Smotherman et al., 2020). In total, we analyzed the activity of 165 neurons recorded from throughout the tonotopic axis of primary auditory cortex (A1) in four bats. In addition to the sequences, each neuron was probed with changing sound pressure level and frequency to calculate the frequency response area. In each neuron, we measured the characteristic frequency (CF) and the mean first spike latency (FSL) at the CF $10 \mathrm{~dB}$ above the minimum threshold. The topographical organization of the CF (tonotopy) and the mean FSL of the A1 is provided in Figure 2. As described before (Macias et al., 2020), the A1 of the freetailed bat is organized tonotopically with higher frequencies represented at rostral positions and a 
descendent gradient in the rostro-caudal direction (Figure 2a). The neuronal mean FSL shows an inverse relationship with cortical locations, where shorter latencies are represented more rostrally and longer latencies are represented more in more caudal positions (Figure 2b). Thus, there is an inverse relationship between CF and mean FSL in the A1 of the free-tailed bat (Figure 2c).

The time course of the response, as characterized by the number of spikes per stimulus during each sequence, was modulated by the pulse-echo repetition rate of the sequences. Figure 3 shows a neuron tuned to $35 \mathrm{kHz} \mathrm{CF}$ (see frequency response are in Figure 3a) responding to the three different presentation rates of pulse-echo pairs reflected on the flat surface (blue dots) and the 150 grit (red dots). The response of this exemplary neuron to the $10 \mathrm{~Hz}$ sequence, as in all 165 recorded neurons, did not show a time-dependent change in the number of spikes evoked by each individual pulse-echo combination throughout the sequence. However, there was a progressive decrease in the number of spikes per stimulus across time in the responses to the 12 and $15 \mathrm{~Hz}$ sequences (Figure 3b). The total number of spikes evoked by each sequential pulse-echo stimulus for this neuron in response to the three sequences for each surface are represented in Figure $3 \mathrm{c}$. There was no change in the number of spikes in response to the $10 \mathrm{~Hz}$ sequence. However, repetition rates of both 12 and $15 \mathrm{~Hz}$ produced a significant decrease in the responses per pulse-echo across time, reaching a maximum effect within the first 5 pulses.

Surprisingly, the repetitive presentation of pulse-echo pairs at 12 and $15 \mathrm{~Hz}$ did not change the mean FSL. We calculated the mean FSL in response to each pair in the sequences and plotted these as a function of time (Figure 3d). Note that this neuron's response to the echoes from the 60-grit sandpaper (red dots in Figure 3b and red line in Figure 3d) had a longer response latency relative to the response to the flat spectrum (blue line). The longer latency at this frequency is a 
consequence of the amplitude notch at $35 \mathrm{kHz}$ coinciding with the cells $\mathrm{CF}$, thereby encoding the lower sound pressure level at this frequency (Macias et al., 2020).

Stimulus repetition rates of 12 and $15 \mathrm{~Hz}$ also had an effect on the latency stability (Figure 3e). We calculated the standard deviation of the FSL for each pulse-echo pair over the 30 trials ("SD FSL") to estimate the temporal stability of the FSL response onset. When stimulating with the 10 Hz sequence, we saw no changes in the latency stability across time. However, in response to 12 and $15 \mathrm{~Hz}$ repetition rates we observed a decrease in the SD FSL, indicating a progressive increase in response first spike-time consistency during the stimulus sequence. As a measure of the temporal precision of the response to each pulse-echo, we calculated the half-width half height (HWHH) of the autocorrelogram of the PSTH. The time course of the HWHH for this neuron is plotted in Figure 3f. In response to the $10 \mathrm{~Hz}$ sequences, there are no changes in the HWHH across time. However, when repetition rate is increased to 12 and $15 \mathrm{kHz}$, there is a progressive decrease in the HWHH.

The changes in the number of spikes across time in the example neurons shown in Figure 3 were observed in the remaining 164 recorded cortical neurons (see Figure 4a-c for the neuronal population). To evaluate if the trend to decrease the number of spikes across time was statistically significant, we used the Mann-Kendall test (MKt). In this, a $\mathrm{H}$ value equal to 0 indicates no significant trend and a value of 1 indicates a significant increase or decrease. Overall, there was no change in the number of spikes in response to the $10 \mathrm{~Hz}$ sequence (MKt, $\mathrm{H}=0, \mathrm{p}=0.37$ ). However, repetition rates of both 12 and $15 \mathrm{~Hz}$ produced a significant decrease in the responses per pulse-echo across time (MKt, $\mathrm{H}=1, \mathrm{p}=0.013$ and $\mathrm{p}=0.0022$, respectively).

The effect of the stimulus repetition rates of 12 and $15 \mathrm{~Hz}$ on the latency stability on the neuronal population is shown in Figure 4d-f. When stimulating with the $10 \mathrm{~Hz}$ sequence, we saw no 
changes in the latency variability across time $(\mathrm{MKt}, \mathrm{H}=0, \mathrm{p}=0.474)$. However, in response to 12 and $15 \mathrm{~Hz}$ repetition rates we observed a decrease in the SD FSL (MKt, $\mathrm{H}=1, \mathrm{p}=0.0004$ and $\mathrm{p}=0.0002$, respectively). We observed a similar result for the temporal precision evaluated by the HWHH of the PSTH autocorrelogram (Figure 4g-i). We found no variation of the response precision across time for the response to the $10 \mathrm{~Hz}$ sequences $(\mathrm{MKt}, \mathrm{H}=0, \mathrm{p}=0.27)$ and an increase in the responses to the 12 and $15 \mathrm{~Hz}(\mathrm{MKt}, \mathrm{H}=1, \mathrm{p}=0.0026$ and $\mathrm{p}=0.0011$, respectively).

\section{Fewer spikes improves signal to noise ratio in neuronal synchronization profiles}

As described previously (Macias et al., 2020), a longer response latency is produced when an amplitude notch is present in the echo spectrum at the neuron's CF. This results in the neuron spiking in synchrony with neurons tuned to lower CFs and can thereby create a signature synchronization pattern across A1 representing the spectral envelope of a discreet echo and indirectly the physical properties (i.e. texture) of the reflecting object (Macias et al., 2020). We analyzed whether the decrease in number of spikes per echo together with the increase in response precision influenced the emergent spike synchronization patterns across A1. To do this, we reconstructed the topographical population dynamics from the CF-specific individual responses to each of the pulse-echo combinations and then calculated the corresponding spike synchronization matrices for all neurons. This allowed an evaluation of the changes in synchronization across time during the response to the different stimulus repetition rates. We evaluated spike train synchrony by using the spike-synchronization index (c), which quantifies the degree of synchrony from the relative number of quasi-simultaneous appearances of spikes (Kreuz et al., 2015; Satuvuori et al., 2017; Macias et al., 2020). Each matrix was calculated for 
each trial and from that, we calculated the evoked mean synchronization matrix for the responses to each pulse-echo pair.

The synchronization matrices calculated for all 165 neurons responding to the first and the tenth pulse-echo pair of the flat surface at all three presentation rates are represented in Figure 5a. In the synchronization matrices calculated for the response to the first stimulus at each repetition rates, there was greater synchrony $(0.5-0.8)$ between neurons with the same characteristic frequency and, as expected, fewer instances of synchronous firing $(0-0.5)$ between neurons with different CF. During the presentation at $10 \mathrm{~Hz}$, the synchrony values did not change throughout the pulse-echo sequences. However, at the 12 and $15 \mathrm{~Hz}$ repetition rates the synchronization rate between neurons with different CFs decreased over time, from $<0.5$ to $<$ 0.2 owing to fewer overall spikes and spurious coincidences, while the synchrony between neurons with same CF remained unaffected. Thus, the flat-spectrum echo became more clearly represented in the temporally coded population dynamics as stimulation rate increased.

In the synchronization matrices calculated for the responses to the 60 and 150 grit echo mimics there was an increase in synchrony between neurons tuned to the interference notches and those tuned to lower CFs (Figure 5a and b), creating distinctive and prominent synchronization maps for each sandpaper echo. Background synchrony values continued to diminish throughout the sequence, leading to a steady increase in the signal to noise ratio (SNR) of the matrices. We quantified this by considering each matrix as an image and computed the corresponding SNR, in decibels, using the matrix calculated for the response to the first pulse-echo pair as a reference (Sage and Unser, 2003; Gonzalez and Woods, 2008). The time course of changes in SNR for each surface and each repetition rate are shown in Figure 5d. For all surfaces, there were no changes in SNR in the sequences with $10 \mathrm{~Hz}$ repetition rate, but there was an increase in the 
SNR across time at 12 and $15 \mathrm{~Hz}$. This indicates that the decrease in mean spike numbers accompanied by an increase in spike-timing precision produced higher resolution synchronization maps, supporting better perceptual discrimination of echo spectral envelopes.

\section{Higher call rates provide more information about spectral details.}

To assess how changes in response precision influenced the amount of information carried by the mean FSL about the surface structure, we computed the mutual information (MI) between each individual pulse-echo pair and their respective neural responses. All analyses were based on a post-stimulus window of $50 \mathrm{~ms}$ and each response window was subdivided into $2 \mathrm{~ms}$ bins. We evaluated the performance of the bias-correction methods used to calculate information values, by generating data with statistics close to the real experimental data and estimated the information in the neural codes following procedures used in previous studies (Panzeri et al., 2001; Macias et al., 2020). For each post-stimulus window, information was underestimated when fewer than 16 trials were used (Figure 6). However, considering the number of trials used in our recordings (30), the bias is small and does not affect the MI calculation. To calculate MI for individual neurons, we grouped the neurons according to their $\mathrm{CF}$ and its relationship to the frequency of the amplitude notches derived from the sandpaper interference patterns. Neurons were clustered in four groups (non-notched, 30,35 and $45 \mathrm{kHz}$ ). Non-notched refers to those neurons where the CF did not overlap in frequency with any echo amplitude notch. The frequency response areas of four example neurons are depicted in Figure 7. In this example neuron as well as in the remaining units, there were no changes in MI across time in response to any of the sequences for the non-notched group (Figure 7a). In the remaining sets (Figure 7b-d), there was no change in the $\mathrm{MI}$ in response to the $10 \mathrm{~Hz}$ sequence, however, repetition rates of 12 and $15 \mathrm{~Hz}$ produce an increase in MI across time. Our MI calculations demonstrate that 
increasing pulse repetition rate would help an external observer more reliably classify and identify the reflecting object surface that created the echo. The reasons for this were two-fold: reduced noise and increased latency stability and spike time precision. The reduced firing rate that occurred at higher stimulus repetition rates due to forward suppression paradoxically leads to more information about fine acoustic features embedded in the echo, which in this case relates to the target object's shape.

\section{Discussion}

This study characterized how the temporal pattern of acoustic stimuli affected biosonar information coding in the bat primary auditory cortex. Bats and cetaceans emit very brief broadband signals at high repetition rates to perform echolocation, one of the fastest and most precise examples of sensorimotor integration known for vertebrates (Grinnell, 1989). During flight, bats actively modulate their pulse emission rates, and here we show that in the auditory cortex, neuronal response dynamics were very sensitive to pulse repetition rate, with the main effect being fewer but more temporally precise action potentials occurring at higher repetition rates. Surprisingly, faster repetition rates had no impact on cortical neuron first-spike latency, instead producing an increase in the spike temporal precision and consequently an increase in MI about stimulus identity. Reduced overall firing at the population level lowered the signal to noise ratio across the neuronal ensemble synchronization patterns and, hence, comprehensively supported better auditory object discrimination.

The forward suppression of auditory neurons that occurs in response to repeated stimulation is thought to result from a combination of synaptic depression and engagement of inhibitory circuits in A1 (Wehr and Zador, 2005; Bayazitov et al., 2013). This is generally thought to 
create a sensitivity for novel stimuli and context by suppressing responses to sustained background stimulation. Some of the proposed functional roles for forward suppression are that it contributes to cortical gain control (Ohzawa et al., 1982), enhances stimulus discriminability (Müller et al., 1999), maximizes information transmission by matching the coding strategy to stimulus statistics (Fairhall et al., 2001) and emphasizes new and interesting sounds (Ulanovsky et al., 2003; Malmierca et al., 2009). Reduction or total suppression of the cortical neuronal response at high repetition rates has been described not only in bats but also in rodents, birds and monkeys (Amin et al., 2004; Bartlett and Wang, 2005; Wehr and Zador, 2005; Narayan et al., 2007; Bayazitov et al., 2013; Schneider and Woolley, 2013; Zhou and Wang, 2014; Beetz et al., 2016; Hechavarría et al., 2016). In rats, cats and monkeys the neuronal response in the auditory cortex may be completely suppressed (Ulanovsky et al., 2004; Wehr and Zador, 2005; Bayazitov et al., 2013). Similarly, in response to natural acoustic sequences, neurons in the auditory cortex of the fruit-eating bat Carollia perspicillata showed an initial response to the first acoustic elements before they were strongly suppressed (Beetz et al., 2016; Hechavarría et al., 2016). In Carollia it was though that this suppression allowed for a more precise extraction of target distance information (Beetz et al., 2016), acting as a physiological filter that operates in the time domain to ensure sharp target-distance tuning and a more distinct topographic organization of echo delays. Furthermore, the receptive fields of target-range (pulse-echo delay-tuned) neurons in fruit bat auditory cortex became sharper with increasing repetition rate (O'Neill and Suga, 1982; Wong et al., 1992; Tanaka and Wong, 1993). Recent computational models of animal biosonar proposed that the bat auditory cortex is likely to encode echo spectral details by transposing amplitude-latency trade-offs in the ascending auditory system into a topographical profile of spike time-registrations (Simmons, 2012; Ming et al., 2021). Here, we show that in 
insectivorous bats, the forward suppression induced by increasing pulse repetition rate sharpened the resolution of these time registrations, and thereby enhanced the cortical ensemble representation of echo spectral envelope, which may be particularly important for bats hunting insects on the wing.

During an attack on a flying insect or while approaching an obstacle, bats increase their pulse emission rates (Griffin, 1958; Schnitzler and Kalko, 2001; Ratcliffe, 2009). Increasing call emission rates generates more frequent information updates about the structure and position of the target (Moss and Surlykke, 2001; Ratcliffe, 2009; Moss and Surlykke, 2010; Elemans et al., 2011). In these experiments we found that as pulse emission rate increased, the auditory cortical substrate captured more information from each echo in the form of spike times about the reflecting surface structure. This suggests that when echolocating bats increase pulse repetition rate, they not only increase the rate of information flow but also the quality of that information by sharpening the cortical representation of the echo spectrum (Ulanovsky et al., 2003; Malmierca et al., 2009). The primary auditory cortex in the bat leverages the effects of forward suppression to improve their discrimination of auditory objects representing physical objects of different shape and structure.

Our experiments were conducted under pentobarbital anesthesia. Pentobarbital is known to decrease spontaneous activity in the cortex through a facilitation of inhibitory postsynaptic potentials which may accelerate or enhance forward suppression dynamics. However, the mean time course of forward suppression observed in these experiments was similar to that described in awake primates (Fishman et al., 2001; Micheyl et al., 2005; Zhou and Wang, 2014; Malone et al., 2015). Recordings of neural discharges from awake guinea pigs (Creutzfeldt et al., 1980) (Creutzfeldt et al. 1980) and recordings of intracortical slow-wave activity in cats (Etholm et al., 
1976) have documented that a significant amount of forward inhibition contributes to response dynamics in the awake preparation. Furthermore, Hechavarría et al. (2016) reported that they found similar suppression dynamics in the AC of both anesthetized and awake bats. Nonetheless, our interpretation of the temporal response characteristics of cortical cells obtained in this study must be presumed to include contributions from anesthesia. In addition, our observations in anesthetized animals exclude the possibility of the role played by attention when animals are actively producing sounds. Further experiments will be required to disentangle the contributions of pentobarbital from the stimulus-driven inhibitory effects described here. Although recordings in an actively vocalizing bat would be most ideal to fully understand the neuronal response properties of the auditory cortex, the present study still offers valuable new insights on how temporal arraignment of sounds affects the cortical circuits processing behaviorally relevant stimuli.

\section{References}

Amin N, Grace J, Theunissen FE (2004) Neural response to bird's own song and tutor song in the zebra finch field L and caudal mesopallium. J Comp Physiol A 190:469-489.

Bartlett EL, Wang X (2005) Long-lasting modulation by stimulus context in primate auditory cortex. J Neurophysiol 94:83-104.

Bayazitov IT, Westmoreland JJ, Zakharenko SS (2013) Forward suppression in the auditory cortex is caused by the Ca_v_3.1 calcium channel-mediated switch from bursting to tonic firing at thalamocortical projections. J Neurosci 33:18940-18950.

Beetz MJ, Hechavarría JC, Kössl M (2016) Temporal tuning in the bat auditory cortex is sharper when studied with natural echolocation sequences. Scientific Rep 6:1-13. 
Brosch M, Schreiner CE (1997) Time course of forward masking tuning curves in cat primary auditory cortex. J Neurophysiol 77:923-943.

Creutzfeldt O, Hellweg F-C, Schreiner C (1980) Thalamocortical transformation of responses to complex auditory stimuli. Exp Brain Res 39:87-104.

Eggermont JJ (1992) Stimulus induced and spontaneous rhythmic firing of single units in cat primary auditory cortex. Hear Res 61:1-11.

Elemans CP, Mead AF, Jakobsen L, Ratcliffe JM (2011) Superfast muscles set maximum call rate in echolocating bats. Science 333:1885-1888.

Etholm B, Gjerstad L, Skrede K (1976) Size and duration of inhibition in the medial geniculate body in unanesthetized cats. Acta oto-laryngologica 81:102-112.

Fairhall AL, Lewen GD, Bialek W, van Steveninck RRdR (2001) Efficiency and ambiguity in an adaptive neural code. Nature 412:787-792.

Firzlaff U, Schuller G (2007) Cortical responses to object size-dependent spectral interference patterns in echolocating bats. Eur J Neurosci 26:2747-2755.

Fishman YI, Reser DH, Arezzo JC, Steinschneider M (2001) Neural correlates of auditory stream segregation in primary auditory cortex of the awake monkey. Hear Res 151:167187.

García-Rosales F, Beetz MJ, Cabral-Calderin Y, Kössl M, Hechavarria JC (2018) Neuronal coding of multiscale temporal features in communication sequences within the bat auditory cortex. Comm Biol 1:1-14.

Gonzalez R, Woods R (2008) Digital image processing: Pearson prentice hall. Upper Saddle River, NJ 1:376-376.

Griffin DR (1958) Listening in the dark: the acoustic orientation of bats and men. 
Grinnell AD (1989) Listening to the voice within. Nature 341:488-489.

Habersetzer J, Vogler B (1983) Discrimination of surface-structured targets by the echolocating bat Myotis myotis during flight. J Comp Physiol A 152:275-282.

Hechavarría JC, Beetz MJ, Macias S, Kössl M (2016) Vocal sequences suppress spiking in the bat auditory cortex while evoking concomitant steady-state local field potentials. Scientific Rep 6:1-15.

Hörpel SG, Firzlaff U (2019) Processing of fast amplitude modulations in bat auditory cortex matches communication call-specific sound features. J Neurophysiol 121:1501-1512.

Ince RA, Panzeri S, Kayser C (2013) Neural codes formed by small and temporally precise populations in auditory cortex. J Neurosci 33:18277-18287.

Kreuz T, Mulansky M, Bozanic N (2015) SPIKY: a graphical user interface for monitoring spike train synchrony. J Neurophysiol 113:3432-3445.

Macias S, Bakshi K, Smotherman M (2019) Laminar organization of fm direction selectivity in the primary auditory cortex of the free-tailed bat. Front Neural Circ 13.

Macias S, Bakshi K, Garcia-Rosales F, Hechavarria JC, Smotherman M (2020) Temporal coding of echo spectral shape in the bat auditory cortex. PLoS Biol 18:e3000831.

Magri C, Whittingstall K, Singh V, Logothetis NK, Panzeri S (2009) A toolbox for the fast information analysis of multiple-site LFP, EEG and spike train recordings. BMC Neurosci 10:81.

Malmierca MS, Cristaudo S, Pérez-González D, Covey E (2009) Stimulus-specific adaptation in the inferior colliculus of the anesthetized rat. J Neurosci 29:5483-5493.

Malone BJ, Beitel RE, Vollmer M, Heiser MA, Schreiner CE (2015) Modulation-frequencyspecific adaptation in awake auditory cortex. J Neurosci 35:5904-5916. 
Micheyl C, Tian B, Carlyon RP, Rauschecker JP (2005) Perceptual organization of tone sequences in the auditory cortex of awake macaques. Neuron 48:139-148.

Ming C, Haro S, Simmons AM, Simmons JA (2021) A comprehensive computational model of animal biosonar signal processing. PLoS Comp Biol 17:e1008677.

Moss CF, Surlykke A (2001) Auditory scene analysis by echolocation in bats. J Acout Soc Am 110:2207-2226.

Moss CF, Surlykke A (2010) Probing the natural scene by echolocation in bats. Front Behav Neurosci 4:33.

Müller JR, Metha AB, Krauskopf J, Lennie P (1999) Rapid adaptation in visual cortex to the structure of images. Science 285:1405-1408.

Narayan R, Best V, Ozmeral E, McClaine E, Dent M, Shinn-Cunningham B, Sen K (2007) Cortical interference effects in the cocktail party problem. Nat Neurosci 10:1601-1607.

Nelken I, Chechik G, Mrsic-Flogel TD, King AJ, Schnupp JW (2005) Encoding stimulus information by spike numbers and mean response time in primary auditory cortex. J Comp Neurosci 19:199-221.

O'Neill WE, Suga N (1982) Encoding of target range and its representation in the auditory cortex of the mustached bat. J Neurosci 2:17-31.

Ohzawa I, Sclar G, Freeman R (1982) Contrast gain control in the cat visual cortex. Nature 298:266-268.

Panzeri S, Petersen RS, Schultz SR, Lebedev M, Diamond ME (2001) The role of spike timing in the coding of stimulus location in rat somatosensory cortex. Neuron 29:769-777.

Quiroga RQ, Nadasdy Z, Ben-Shaul Y (2004) Unsupervised spike detection and sorting with wavelets and superparamagnetic clustering. Neural Comp 16:1661-1687. 
Ratcliffe JM (2009) 11 Predator-Prey Interaction in an Auditory World. Cognitive ecology II:201.

Sage D, Unser M (2003) Teaching image-processing programming in Java. IEEE Signal Processing Magazine 20:43-52.

Saillant PA, Simmons JA, Dear SP, McMullen TA (1993) A computational model of Echo processing and acoustic imaging in frequency-modulated echolocating bats: The spectrogram correlation and transformation receiver. J Acoust Soc Am 94:2691-2712.

Sanderson MI, Simmons JA (2002) Selectivity for echo spectral interference and delay in the auditory cortex of the big brown bat Eptesicus fuscus. J Neurophysiol 87:2823-2834.

Satuvuori E, Mulansky M, Bozanic N, Malvestio I, Zeldenrust F, Lenk K, Kreuz T (2017) Measures of spike train synchrony for data with multiple time scales. J Neurosci Met 287:25-38.

Schmidt S (1988) Evidence for a spectral basis of texture perception in bat sonar. Nature $331: 617-619$

Schmidt S (1992) Perception of structured phantom targets in the echolocating bat, M egadermalyra. J Acoust Soc Am 91:2203-2223.

Schneider DM, Woolley SM (2013) Sparse and background-invariant coding of vocalizations in auditory scenes. Neuron 79:141-152.

Schnitzler H-U, Kalko EK (2001) Echolocation by insect-eating bats. Bioscience 51:557-569.

Schnupp JW, Hall TM, Kokelaar RF, Ahmed B (2006) Plasticity of temporal pattern codes for vocalization stimuli in primary auditory cortex. J Neurosci 26:4785-4795. 
Simmons J, Lavender W, Lavender B, Doroshow C, Kiefer S, Livingston R, Scallet A, Crowley D (1974) Target structure and echo spectral discrimination by echolocating bats. Science 186:1130-1132.

Simmons JA (2012) Bats use a neuronally implemented computational acoustic model to form sonar images. Curr Op Neurobiol 22:311-319.

Simmons JA, Chen L (1989) The acoustic basis for target discrimination by FM echolocating bats. J Acoust Soc Am 86:1333-1350.

Simmons JA, Moss CF, Ferragamo M (1990) Convergence of temporal and spectral information into acoustic images of complex sonar targets perceived by the echolocating bat, Eptesicus fuscus. J Comp Physiol A 166:449-470.

Smotherman M, Odunsi S, Hobbs M, Croft T (2020) Acoustic discrimination of fine surface textures by echolocating free-tailed bats. J Acoust Soc Am 148:2556-2556.

Suga N (1989) Principles of auditory information-processing derived from neuroethology. J Exp Biol 146:277-286.

Tanaka H, Wong D (1993) The influence of temporal pattern of stimulation on delay tuning of neurons in the auditory cortex of the FM bat, Myotis lucifugus. Hear Res 66:58-66.

Ulanovsky N, Las L, Nelken I (2003) Processing of low-probability sounds by cortical neurons. Nat Neurosci 6:391-398.

Ulanovsky N, Las L, Farkas D, Nelken I (2004) Multiple time scales of adaptation in auditory cortex neurons. J Neurosci 24:10440-10453.

Wehr M, Zador AM (2005) Synaptic Mechanisms of Forward Suppression in Rat Auditory Cortex. Neuron 47:437-445. 
Wong D, Maekawa M, Tanaka H (1992) The effect of pulse repetition rate on the delay sensitivity of neurons in the auditory cortex of the FM bat, Myotis lucifugus. Journal of Comparative Physiology A 170:393-402.

Yin P, Johnson JS, O'Connor KN, Sutter ML (2011) Coding of amplitude modulation in primary auditory cortex. Journal of neurophysiology 105:582-600.

Zhou Y, Wang X (2014) Spatially extended forward suppression in primate auditory cortex. European Journal of Neuroscience 39:919-933.

\section{Figure Legends}

Figure 1 Acoustic stimuli.

Oscillogram of the pulse-echo (echo highlighted in blue) pairs and spectrogram and power spectra of the echoes used as stimulus. Insets indicate the frequencies of the amplitude notches in the echoes of surface. The emitted signal was a $3 \mathrm{~ms}$ downward frequency-modulated sweep starting at $70 \mathrm{kHz}$ and ending at $20 \mathrm{kHz}$.

Figure 2. Topographical organization of the characteristic frequency (CF) and the mean first spike latency (mean FSL) in the primary auditory cortex (A1) of the Mexican freetailed bat.

(a) Characteristic frequency as a function of the anterior-posterior location. (b) Mean first spike latency as a function of the cortical location. (c) Relation between CF and mean FSL. Details about the topographical and functional organization of the A1 in the Mexican free-tailed bat can be found in (Macias et al., 2019). 
Figure 3. Example response to repetitive stimuli.

(a) Frequency response area of an example neuron tuned with a characteristic frequency $(\mathrm{CF})$ of $35 \mathrm{kHz}$. The CF coincides with the frequency of one of the amplitude notches in the echo recorded from the 150-grit sandpaper. (b) Dot-raster display of the response of the neuron shown in (a) to three sequences of different repetition rate. In each response, blue dots represent the response to the flat surface and red dots represent the response to the 150 -grit sandpaper. (c) Number of spikes as a function of time for the responses shown in (b). (d) Mean first spike latency (FSL) as a function of time for the responses shown in (b). (e) Latency stability across trials calculated as the Standard Deviation of the mean FSL as a function of time for the responses shown in (b). (f) Response precision calculated as the half-width half height of the autocorrelation function of the PSTH as a function of time.

\section{Figure 4. Responses to repetitive stimuli in the cortical neuronal population.}

(a) In response to the $10 \mathrm{~Hz}$ sequence, the number of spikes per trial did not change across time for any of the three surfaces (flat, 60- and 150-grit). (b-c) There is a reduction of the number of spikes across time in response to the 12 and $15 \mathrm{~Hz}$ sequences for the three surfaces. Red line indicates the mean normalized number of spikes for all 165 neurons. (d) In response to the $10 \mathrm{~Hz}$ sequence, there are no changes in the latency stability across time for any of the three surfaces (flat, 60- and 150-grit). (e-f) There is an increase of the latency stability (reduction of the SD of the FSL) across time in response to the 12 and $15 \mathrm{~Hz}$ sequences for the three surfaces. (g) There were no changes in the response precision (calculated as the half-weight half-height, $\mathrm{HWHH}$, on the autocorrelation function of the PSTH) across time in response to the $10 \mathrm{~Hz}$ sequence. (h-i) Decrease of the HWHH in response to the 12 and $15 \mathrm{~Hz}$ sequences. 
Figure 5. Sequences of pulses-echoes at high repetition rate increase signal to noise ratios of neuronal synchronization patterns.

(a) Synchronization patterns calculated for the responses to the first and the tenth pulse-echo pairs from the flat surface of each repetition rate. (b) Synchronization patterns calculated for the responses to the first and the tenth pulse-echo pairs from the 60 -grit surface of each repetition rate. (c) Synchronization patterns calculated for the responses to the first and the tenth pulseecho pairs from the 150-grit surface of each repetition rate. All synchronization matrices were calculated for 155 neurons from four bats. (d) Signal to noise ratio (SNR) calculated for each synchronization pattern in response to the three sequences. In each plot, black lines represent the response to the flat surface, red line represents the response to the 60 -grit surface and blue line represents the response to the 150-grit surface. SNR was calculated using the response to the first pulse-echo pair in each sequence as a reference.

\section{Figure 6. Mutual information (MI) and the effect of sample size (\# trials).}

Performance for bias-correction method used to calculate information values. Figure shows the data from the responses of neurons tuned to $30 \mathrm{kHz}$. Data were generated with statistics derived from the real experimental data to assess whether the number of trials included was sufficient for accurate calculation of MI). Calculation of MI was accurate at $>15$ trials, which was less than the number (30) used in our study.

\section{Figure 7. Mutual information increases across time.}

(a) Non-notched neuron (CF does not coincide with the frequency of any notch). (b) Example neuron with $\mathrm{CF}=30 \mathrm{kHz}$. (c) Example neuron with $\mathrm{CF}=35 \mathrm{kHz}$. (d) Example neuron with $\mathrm{CF}=45$ $\mathrm{kHz}$. Mutual information across time for each example neuron is represented in blue. Gray lines 
617 represent mutual information across time for all the recorded neurons. Red line indicates mean 618 mutual information for all neurons in each group. 

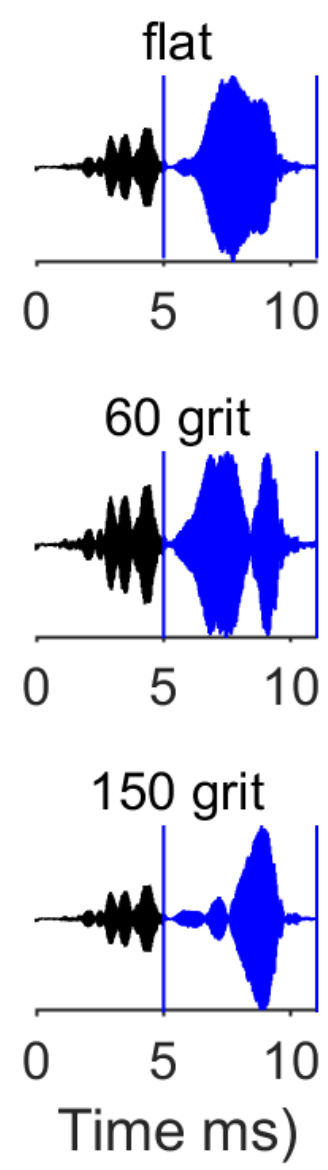
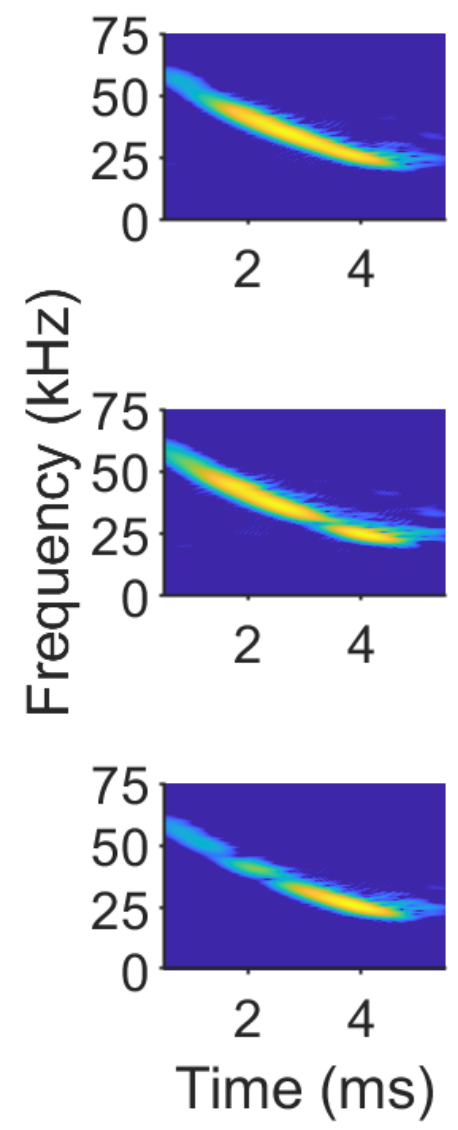
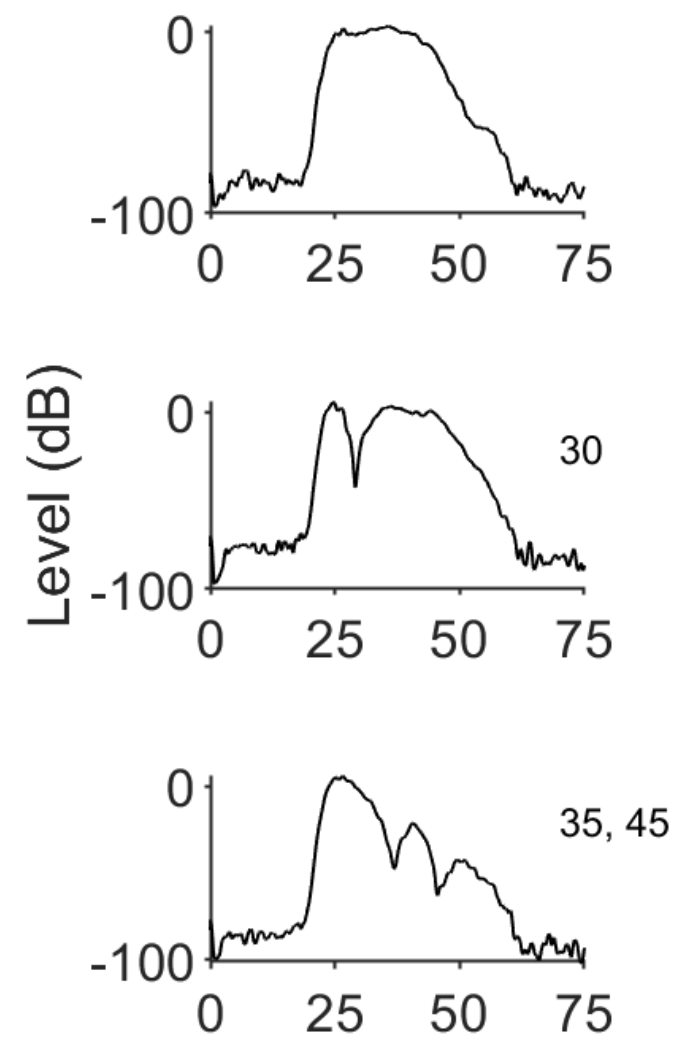

Frequency (kHz) 


\section{a}

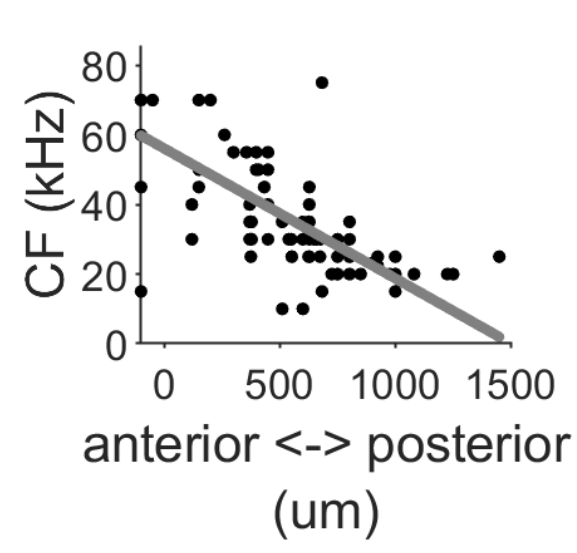

b

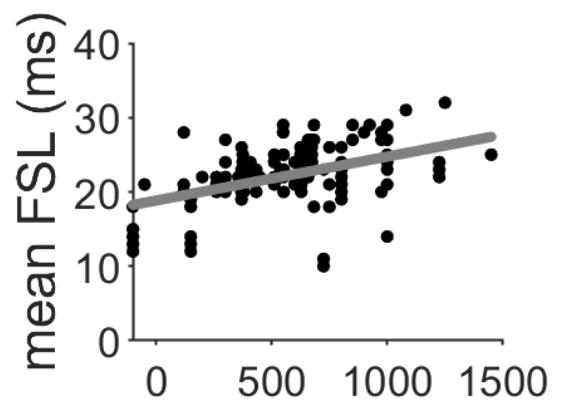
anterior <-> posterior

(um)
C

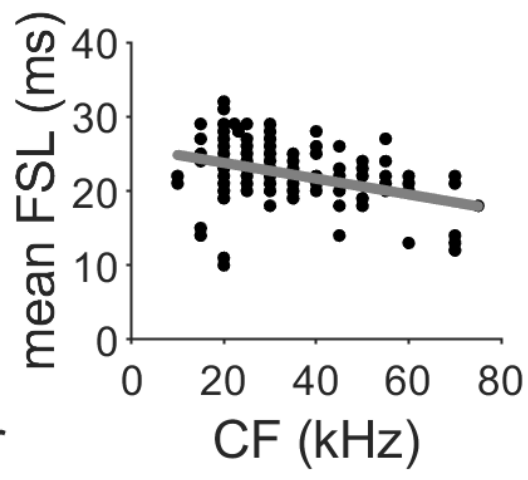



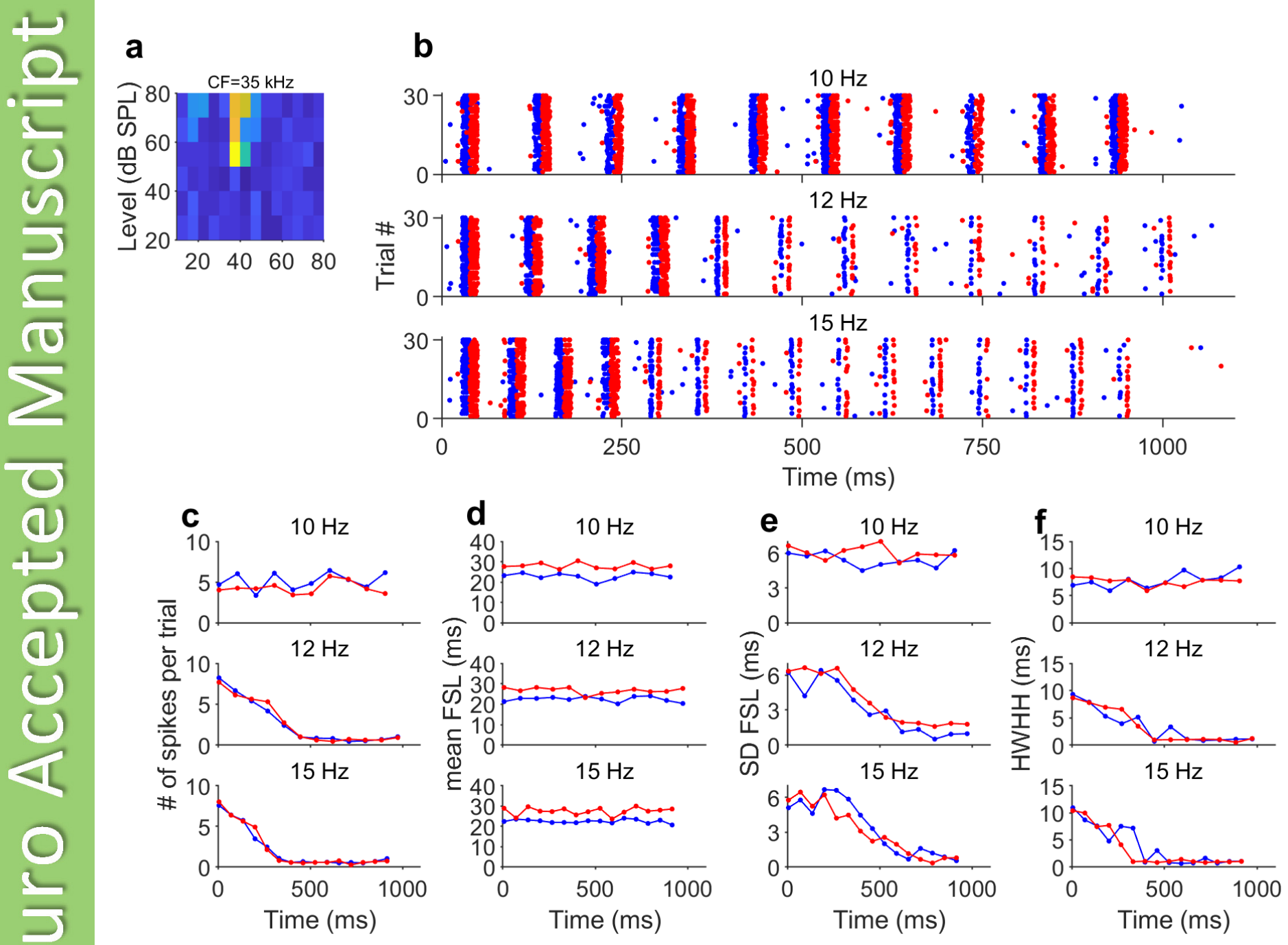

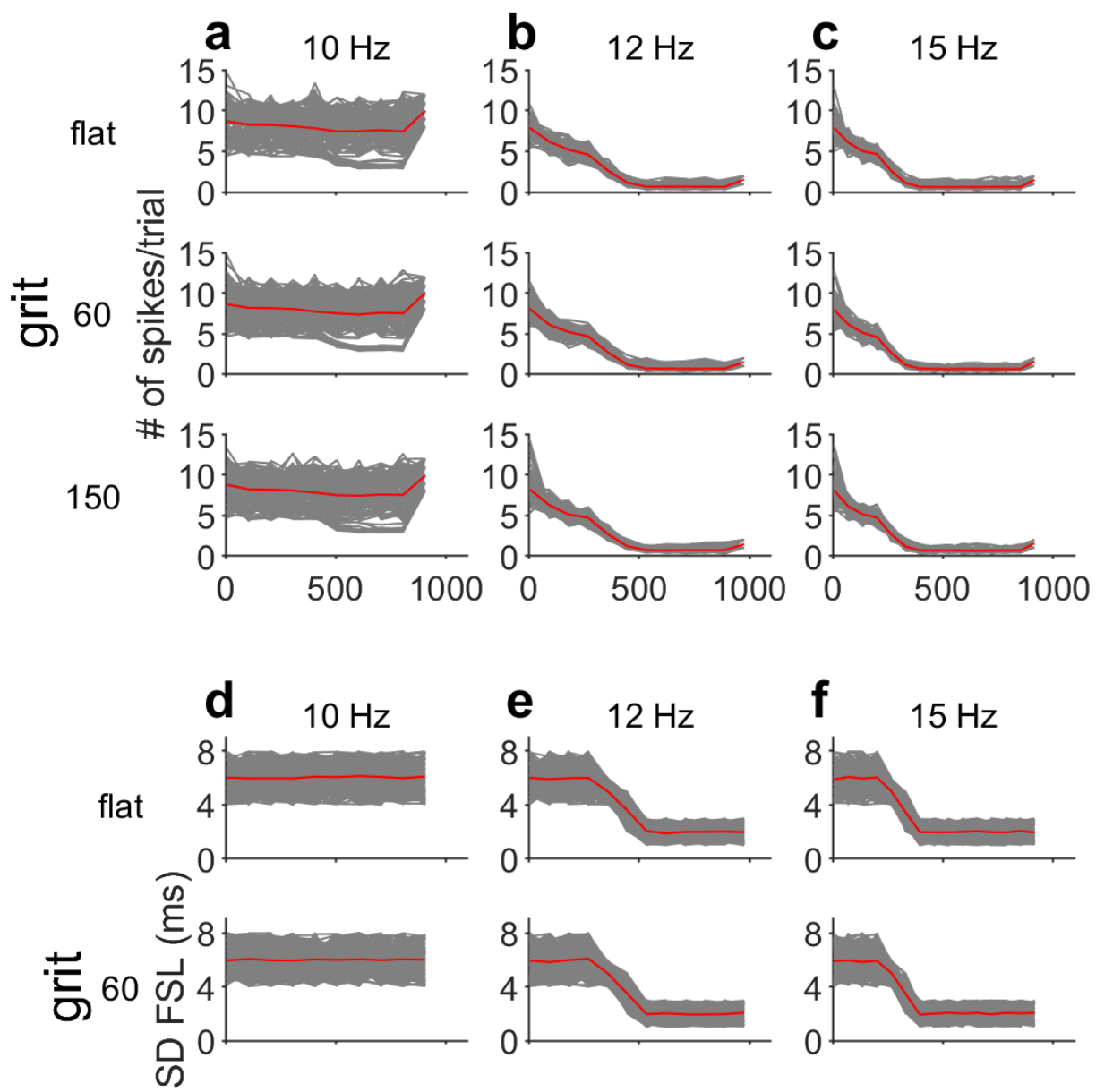

150
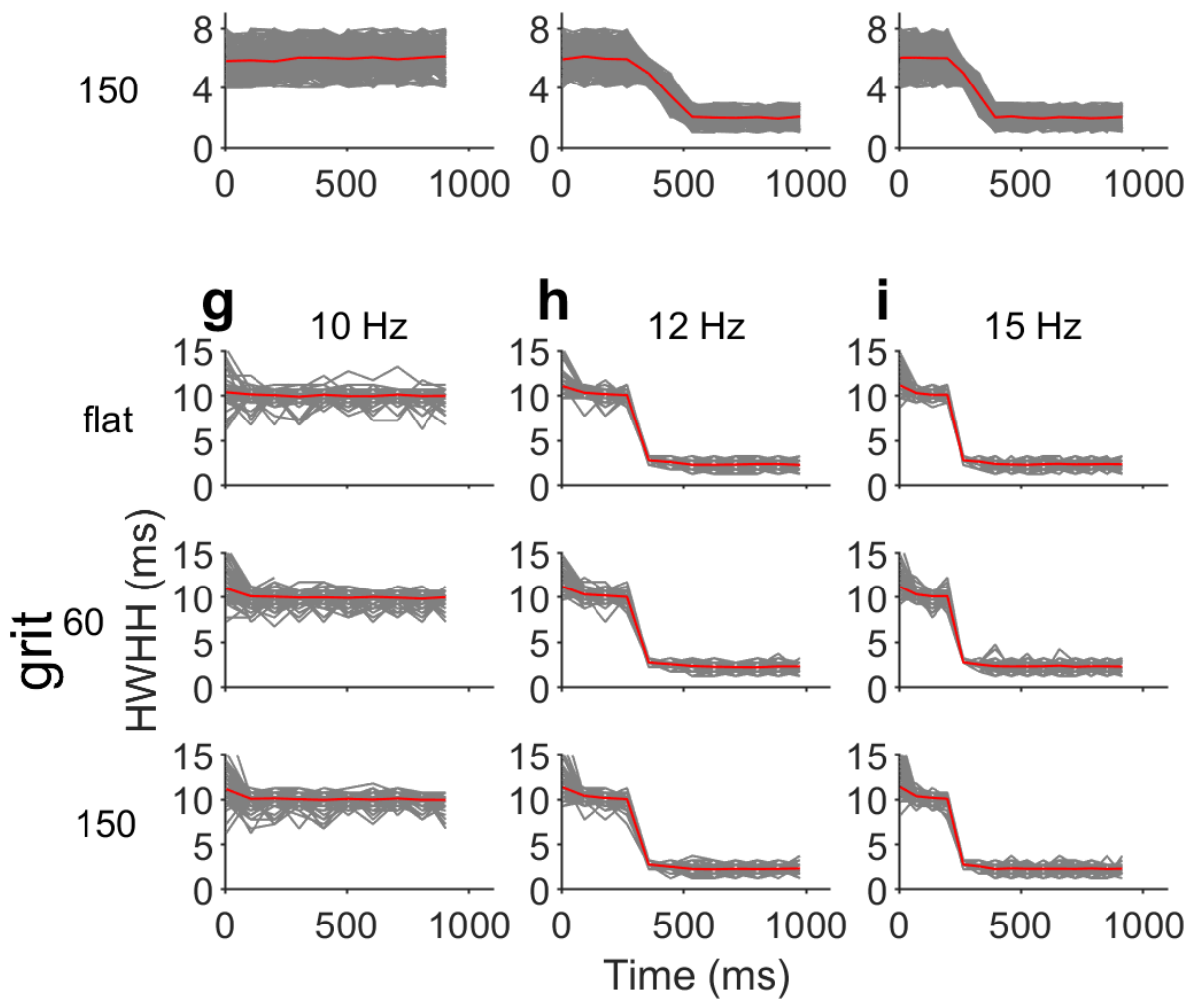

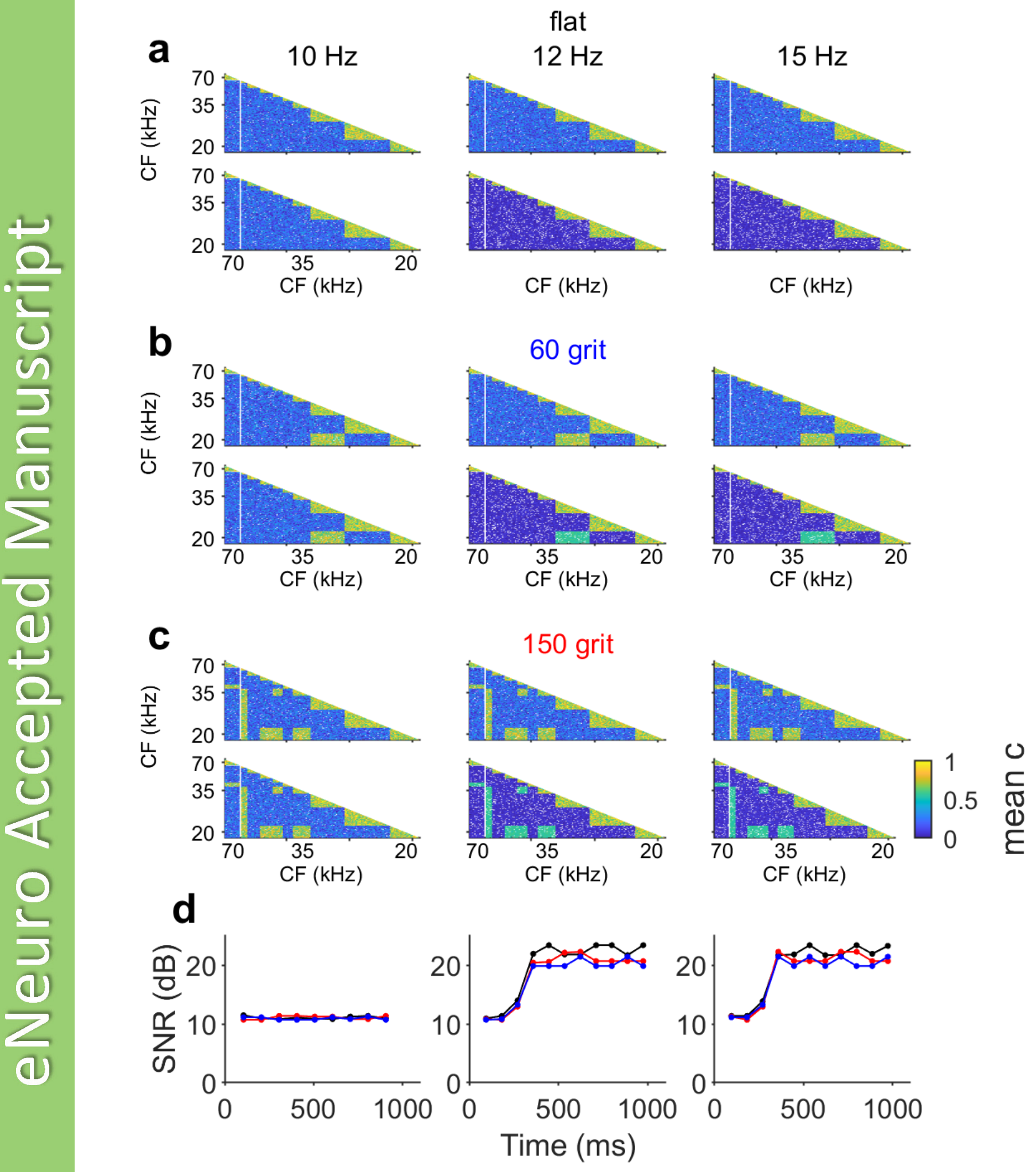


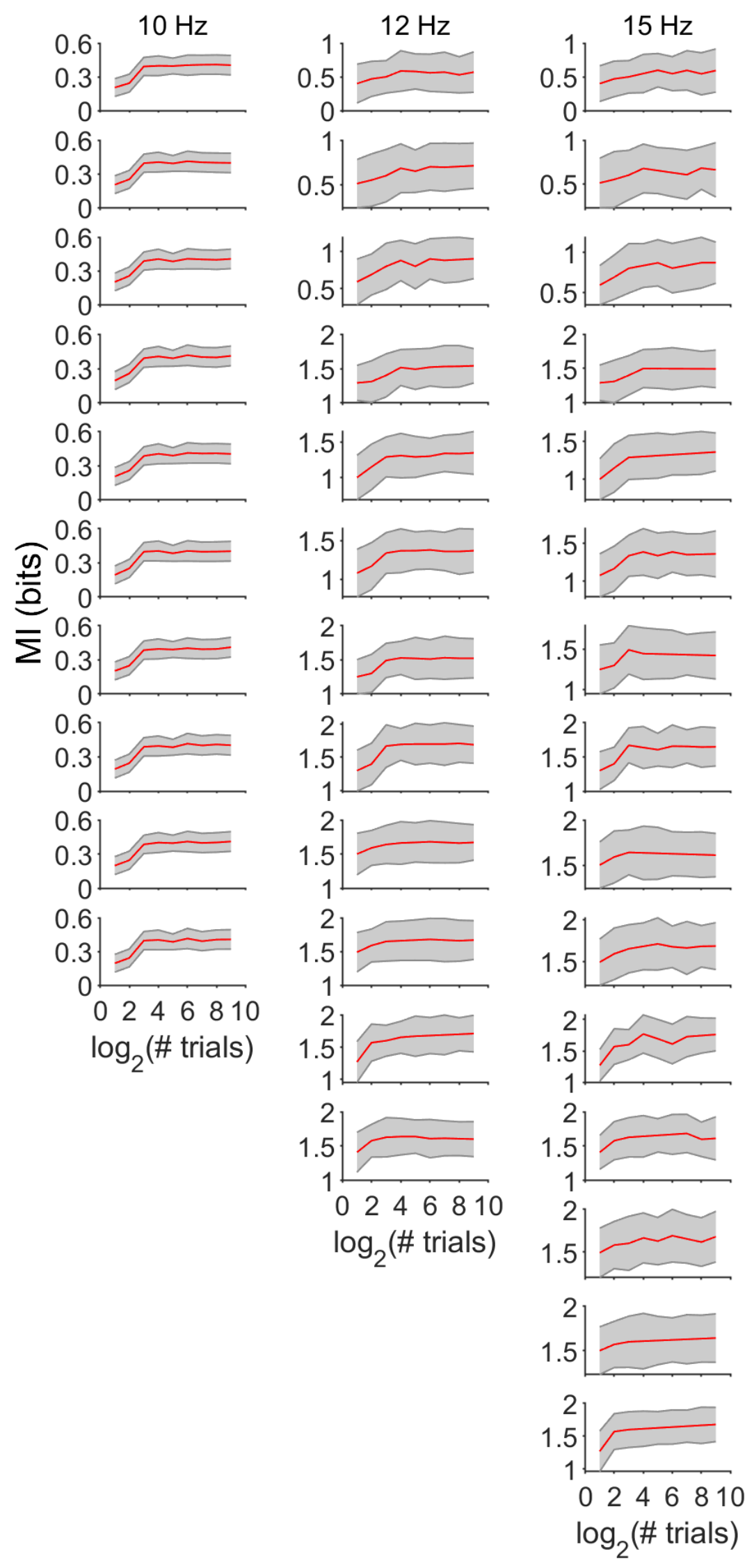



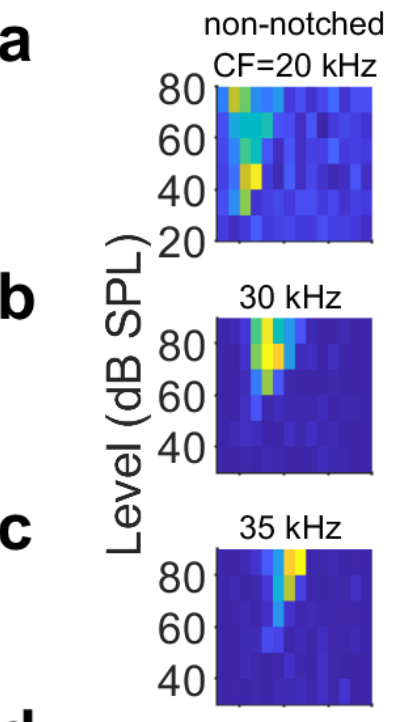

(d)

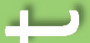

(2)

(1)

d
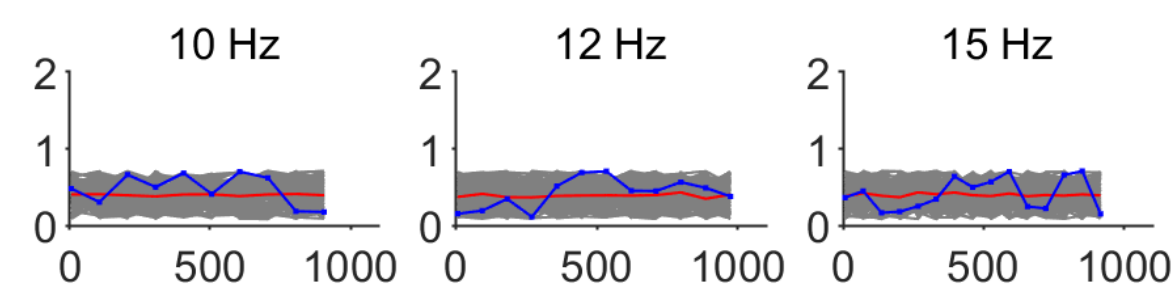

()

(u)

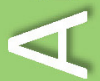
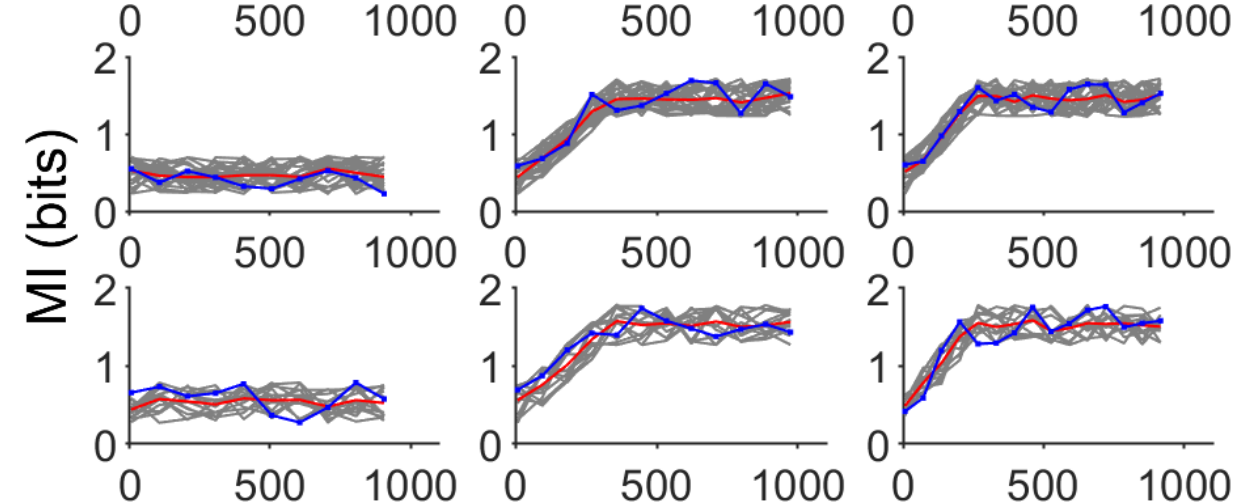

(2) Frequency $(\mathrm{kHz})$
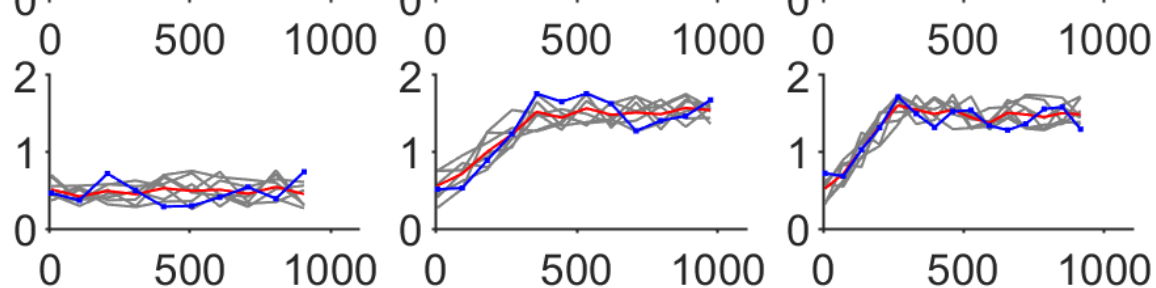

Time (ms) 